\title{
Entre tortugas, canales y árboles talados. Aproximación arqueológica a los procesos industriales manifiestos en Tortuguero, Costa Rica (1871-1950)
}

\section{Between Timbers, Channels and Turtles. Archaeological Approach to Tortugueros's Industrial Processes, Costa Rica (1871-1950)}

\author{
Mónica Aguilar Bonilla* \\ Jeffrey Peytrequín Gómez ${ }^{* *}$
}

\begin{abstract}
Resumen: Como parte de los resultados emanados del proyecto de investigación «Arqueología Industrial: Estado del arte y primer inventario nacional» — código 219-B8-077- de la Universidad de Costa Rica, se presentan los siguientes avances de la temporada de campo 2018 respecto al sitio arqueológico Tortuguero - sigla L-324 Tg - Este trabajo aborda el estudio del pasado reciente - finales del siglo XIX hasta 1970 - de una localidad ubicada al norte del litoral Caribe de Costa Rica, ello a partir de sus restos materiales y desde la perspectiva de la arqueología industrial.
\end{abstract}

Palabras claves: arqueología; arqueología industrial; explotación forestal, patrimonio industrial, recursos forestales, historia económica; Tortuguero; Caribe; Costa Rica.

Abstract: As a part of a current research project's results at University of Costa Rica «Industrial Archaeology: background and first national inventory» — code 219-B8-077-, this article presents 2018 field work advance data related to Tortuguero archaeological site $-\mathrm{L}-324 \mathrm{Tg}$ - and make an approach to this locality recent past — end of XIX century-1970 — situated on Costa Rica's

Fecha de recepción: 20/08/2019 - Fecha de aceptación: 01/01/2020

* Costarricense. M.Sc. en Antropología con énfasis en Arqueología por la Universidad de Costa Rica (UCR), Costa Rica. Docente e investigadora en la Escuela de Antropología de la UCR. Correo electrónico: monica.aguilar@ucr.ac.cr.

** Costarricense. Doctor en Ciencias Sociales por la Universidad Nacional (UNA), Costa Rica. Docente e investigador en la Escuela de Antropología de la Universidad de Costa Rica (UCR), Costa Rica. Correo electrónico: jeffrey.peytrequin@ucr.ac.cr. 
Caribbean northern coast. The above emphasizing the material culture study from an Industrial Archaeology perspective.

Keywords: Archaeology; Industrial Archaeology; Forestry; Industrial Heritage; Forest Resources; Economic History; Tortuguero; Caribbean Coast.

\section{Introducción y contextualización ambiental ${ }^{1}$}

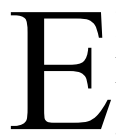
1 sitio arqueológico industrial Tortuguero se encuentra en el cantón de Pococí, distrito de Colorado, provincia de Limón. En el poblado de Tortuguero, localizado en el Caribe Norte costarricense, se desarrolló - desde finales del siglo XIX e inicios del XX - por un lado, la explotación intensiva de las tortugas y la consecuente exportación masiva de su carne y caparazones hacia Inglaterra, ya que en ese destino era un producto muy apetecido; así como la extracción de especies forestales finas, esto último para su distribución en Europa y varios países de América. Esta segunda actividad promovió una mayor alteración del paisaje a partir del siglo XX, lo cual se ve reflejado en la construcción de canales artificiales y la limpieza — draga — de otros canales fluviales naturales.

Debido a la presencia de un importante recurso hídrico en la zona, en Tortuguero se desarrolló la tala aprovechando el transporte rivereño. Los canales existentes fueron utilizados como medio de comunicación y de transporte de los troncos que se cortaban desde los bosques ubicados río arriba y que luego, eran «conducidos» hasta llegar al aserradero. Dicho lugar fue el primer punto de la cadena de exportación, esto, fundamentalmente asociado al actual atracadero de Tortuguero $\mathrm{y}$, desde allí, la madera era dirigida al puerto de Limón. Por esta razón se dio, en la primera mitad del siglo XX, además, la excavación de canales artificiales en sectores clave e inmediatos a los canales naturales; lo anterior para facilitar el traslado de los troncos que se extraían de las fincas más lejanas al recurso hídrico.

La zona donde se asienta la comunidad de Tortuguero, así como el Parque Nacional homónimo, se sitúa en una amplia cuenca de subsidencia, llamada la depresión de Nicaragua, que se extiende desde el Golfo de Fonseca - en el litoral pacífico de Honduras-, por El Salvador, Nicaragua y hasta llegar al litoral Caribe costarricense. En Costa Rica, esta cuenca se conoce como la «Cuenca de Limón». Por su parte, a nivel geomorfológico, el Caribe de Costa Rica se puede dividir en 5 unidades, de las cuales están presentes en Tortuguero 3: las unidades de conos volcánicos antiguos, las llanuras aluviales y la llanura costera. La

1 Agradecimientos. Al señor Cloyd Taylor Martínez y su familia, vecinos de la localidad de Tortuguero, por compartir su historia. Al arqueólogo Lic. Marco Arce Cerdas, quien colaboró con el levantado de las fotogrametrías en 3D y la realización de las otras imágenes expuestas en este artículo. Su ayuda desinteresada ha sido fundamental para el avance de este proyecto. A la arqueóloga japonesa Waka Kuboyama, quien tradujo la marca y especificaciones mostradas en la bomba Tsuda. Al personal del Parque Nacional Tortuguero, por su colaboración. A la comunidad de Tortuguero, por siempre recibirnos con las puertas abiertas y manifestar un interés genuino por nuestras labores y su historia reciente. 
altura de esta zona comprende desde el nivel del mar - en el límite noreste del parque - hasta los $311 \mathrm{msnm}$ en las Lomas de Sierpe; localizadas en el sector suroeste del Parque Nacional. ${ }^{2}$

El área de estudio se encuentra en la cuenca hidrográfica del río Tortuguero. Los cuerpos de agua más importantes de dicha cuenca - dentro del Parque Nacional - son California, Jalova, Caño Negro, Sérvulo, Sierpe, Tortuguero, Penitencia y Suerte. En Tortuguero predomina el clima tropical húmedo y muy húmedo, la entrada de los vientos alisios del norte y el noreste llevan a la zona mucha humedad en forma de lluvia. En el sector norte, la precipitación promedio anual alcanza los $6000 \mathrm{~mm}$, de julio a diciembre son los meses más lluviosos y marzo, abril y octubre los que presentan menos precipitación. Asimismo, la temperatura promedio anual oscila entre $\operatorname{los} 25^{\circ} \mathrm{C}$ y $\operatorname{los} 30^{\circ} \mathrm{C} .^{3}$

Dado que en este sector del país se dio la extracción de carne de tortuga y la tala masiva de especies forestales, se incluyen los siguientes datos para comprender las razones del porqué de dichas actividades.

El Parque Nacional Tortuguero se encuentra — en su totalidad — dentro de la zona de vida bosque muy húmedo tropical, cuenta con distintas asociaciones vegetales como la litoral, yolillales, bosques pantanosos anegados, bosques de galería, bosque sobre lomas, comunidades herbáceas de laguna y pantanos herbáceos. ${ }^{4}$

Asimismo, presenta el Subsistema Intermareal, el cual — dentro del Parque Nacional - es una franja de costa de $24 \mathrm{~km}$ de playa de arena fina que se ubica desde el límite sur de dicho parque, cubre el sector de Laguna de Jalova y se extiende hasta la comunidad de Barra de Tortuguero. Este subsistema está expuesto — regular y periódicamente - a la acción de las mareas y es allí donde los organismos acuáticos cuentan con adaptaciones para sobrevivir —a niveles de agua y oxígeno variables - por períodos prolongados. El sistema marino del Parque Nacional Tortuguero se clasifica en sistemas y subsistemas, según los regímenes de mareas y la profundidad del océano. ${ }^{5}$

Debido a lo anterior, en la actualidad se conserva una gran diversidad de flora y fauna; sin embargo, y previo a la década de 1970, el lugar era utilizado para la extracción de varias especies, estas tanto para su comercialización a gran escala como para el consumo doméstico.

Dado el peligro que corrían las especies, tras su explotación desmedida por décadas, se estableció el 24 de septiembre de 1970 el Parque Nacional

2 Área de Conservación Tortuguero, «Proyecto Fortalecimiento del Programa de Turismo en Áreas Silvestres Protegidas. Plan de turismo sostenible Parque Nacional Tortuguero PNT» (Guápiles, Limón, sin fecha), 14, disponible en: http://www.sinac.go.cr/ES/transprncia/Planificacin\%20y\%20Gestin\%20BID/Gesti $\%$ C3\%B3n\%20Sostenible\%20del\%20Turismo\%20en\%20ASP/Planes\%20de \%20Turismo\%2010\%20 ASP/Plan\%20de\%20Turismo\%20Sostenible\%20del\%20PN\%20Tortuguero.pdf.

3 Ibíd., 14.

4 Ibíd., 15.

5 Ibíd. 
Tortuguero. Este buscaba conservar un importante conjunto de recursos biológicos y naturales, dentro de lo que se contempla: a) especies de flora y fauna en vías de extinción en el trópico americano; b) muestras de las principales asociaciones vegetales de la vertiente Caribe; c) un sistema de ríos, caños y lagunas naturales de extraordinario valor escénico, recreativo y turístico; y d) la colonia de tortugas verdes - Chelonia mydas - que desova en sus playas, precisamente, este lugar es el destino más importante para su anidación en todo el Caribe. ${ }^{6}$

Unido a lo anterior, el Parque Nacional alberga, al menos, 734 especies de plantas y 442 aves, cerca de 138 especies de mamíferos — que representan 101 géneros y 32 familias - 118 especies de reptiles — 76 géneros y 22 familias-, 58 especies de anfibios - 27 géneros y 11 familias - De igual manera, se protege el hábitat del manatí — Trichechus manatus —, uno de los mamíferos más escasos y amenazados de Costa Rica, así como el ecosistema yolillal — Raphia taedigera -; ecosistema de llanura del Caribe que ha sido degradado por la deforestación e incluye, además, una amplia cobertura marina — 50 284 hectáreasy terrestre - 26653 hectáreas - que forma parte del Humedal Caribe Noreste — según el Decreto n. ${ }^{\circ}$ 1235-A, del 24 de septiembre de 1970 —.?

\section{Tortuguero en clave histórica}

\section{Una trampa mortal para las tortugas por más de tres siglos}

La historia reciente de Tortuguero, desde el período colonial y, fundamentalmente, a partir del siglo XVII, no se puede comprender sin su vínculo con la costa miskita de Nicaragua, lugar que tuvo un protagonismo en la exportación de recursos con destino, primero, hacia las islas del Caribe de habla inglesa, luego, a Europa - donde destaca Inglaterra - y siglos más tarde hacia los Estados Unidos y el oeste de la misma Nicaragua. Para inicios del siglo XX, y más recientemente, se incluyó, entre sus contactos comerciales directos, a Alemania, Japón y otros países industrializados. ${ }^{8}$

Respecto a la costa Miskita, para el siglo XVIII Townsend escribió:

Al regresar a Inglaterra, examiné la naturaleza y extensión de las colonias que produjeron tanta incomodidad a España. La incomodidad surgió [...] del comercio contrabandístico [inglés]; de la comunicación de los ingleses con los misquitos, quienes, en tiempo de guerra, fueron usados para molestar a los españoles; y del recelo de que, por medio de aquéllos, los ingleses pudieran establecerse más tarde de alguna manera, por medio de la fuerza, en el Lago de Nicaragua. Esta colonia fue ciertamente valiosa para Inglaterra, como medio de conexión entre Jamaica y el Continente Hispano [...] La caoba era el principal artículo de su comercio; y la exportación anual

6 Ibíd., 14-15.

7 Ibíd., 15.

8 Bernard Nietschman, Memorias de arrecife Tortuga. Historia natural y económica de las tortugas en el Caribe de América Central (Managua, Nicaragua: Fondo de Promoción Cultural del Banco de América, 1977). 
de esta madera, era alrededor de tres millones de pies. Además de estos artículos, enviaban a Inglaterra cuatro toneladas de concha de tortuga [...]. 1792, II: $423 .{ }^{9}$

Para ese entonces se catalogaba a los miskitos como los mejores «tortugueros» del mundo, quienes, a su vez, controlaban las aguas territoriales de una de las regiones productoras de tortuga más extensas del mundo. Los ingleses, por su parte, organizaron la explotación comercial de la tortuga de mar en el Caribe manteniendo una relación comercial — documentada en más de 200 años-con los miskitos; esta fue de tal magnitud que para el año 1635 una compañía inglesa que operaba en Cayos Miskitos se preocupaba respecto a la posibilidad de que «la tortuga pudiera faltarles a los mosquitos»..$^{10}$

La tortuga fue explotada no solo como alimento de colonos y esclavos de la zona, también los primeros europeos la consumían como antídoto contra el escorbuto $^{11}$ y como un «elixir de virtudes extraordinarias». En las Indias Occidentales la carne de este animal era de gran importancia, inclusive considerada una exquisitez para los colonos ricos. Asimismo, el aceite de tortuga se empleó como sustituto de la manteca, como combustible para lámparas y lubricante. Para mediados del siglo XVII ya se desarrollaba el comercio de tortugas vivas entre las Indias Occidentales y Londres, donde la tortuga verde - Chelonia mydas - era apetecida por las personas de alcurnia; su consumo fue un «símbolo de opulencia victoriana». ${ }^{12}$

George Woodbury sugirió que la tortuga verde, tanto como cualquier otro factor natural atractivo para el comercio, fue responsable de la apertura del mar Caribe y de la concentración de actividades piráticas en esa parte del mundo. ${ }^{13}$ Estos grandes y pesados animales eran fáciles de atrapar, además de abundantes en la zona, con altos aportes nutritivos para el ser humano y — lo más importante de todo - podían ser mantenidos vivos — por semanas enteras- en los climas tropicales antes que hubiese sistemas de refrigeración. ${ }^{14}$ Esto último lo hacía un producto muy rentable en relación con su transporte por largas distancias; lo cual implicaba una cantidad considerable de tiempo.

En vínculo a la comercialización de las tortugas, Parsons en 1954 señalaba que los tortugueros del Mar Caribe eran personas blancas de habla inglesa de las Islas Caimán, «en donde los principales mercados locales de carne de tortuga están en las ciudades negroides de tierra firme tales como Colón en Panamá,

9 Ibíd., 64.

10 Ibíd., 65.

11 Según la Real Academia Española -2019-, el escorbuto — del francés scorbut-es una enfermedad producida por la escasez o ausencia en la alimentación de vitamina $\mathrm{C}$, y caracterizada por hemorragias cutáneas, musculares, por una alteración especial de las encías y por fenómenos de debilidad general.

12 James Parsons, «Una clase de alimento tan delicioso», en: Memorias de arrecife Tortuga. Historia natural y económica de las tortugas en el Caribe de América Central (Managua, Nicaragua: Fondo de Promoción Cultural del Banco de América, 1977a), 66-67.

13 George Woodbury, The great days of Piracy (Londres, Inglaterra: Elek, 1954), 106-107. Ver también: Archie Carr, «The passing of the fleet», en Bulletin American Institute of Biological Sciences, 1954, vol. 4, 17.

14 Parsons, «Una clase de alimento tan delicioso», 67. 
Limón en Costa Rica, Bluefields en Nicaragua y Belice, en donde predominan los protestantes de habla inglesa». ${ }^{15}$

En 1722 se reporta que varios barcos jamaiquinos visitaban anualmente la costa centroamericana para pescar y comprar tortugas - enteras, vivas o solo caparazones - a los indios miskitos. El negocio era muy rentable: 3 hombres y 1 muchacho en una canoa, con solo 2 redes y 1 lanza, podían conseguir 180 tortugas en una temporada, cada una de las cuales brindaba unas 150 libras de carne. ${ }^{16}$ Estas tortugas eran mantenidas en corrales - en aguas poco profundas - frente a los cayos, hasta que se necesitaban sacar de allí para su venta. Además de la tortuga verde viva, y de la carne salada o seca comerciada de esta, las exportaciones de «concha» de carey de la costa alcanzaban un promedio de 6000 a 10 000 libras por año ${ }^{17}$ que, junto al impuesto de exportación respectivo, gravaba la raíz de zarzaparrilla y con este se sufragaban, de sobra, los gastos del gobierno inglés en estos menesteres. ${ }^{18}$

Hacia 1825, John Hale — en su visita al propio Tortuguero - indicó que la tortuga verde era abundante y que se capturaban más de 100 especímenes de este animal por día. Los «indios» estaban acostumbrados a cambiar una tortuga de 100 libras por una botella de ron, o por 2 o 3 yardas de tela de algodón común. Este autor también refirió la exuberancia natural del lugar en cuestión, señalando que se necesitaba un volumen grande - libro o publicación - para dar una descripción completa de las aves, bestias, peces y otros «productos» de estas regiones prolíferas; concibiendo que la variedad de bestias y criaturas aladas de Costa Rica era insuperable a las de cualquier otra parte del mundo. ${ }^{19}$

Así, Tortuguero tuvo un papel fundamental en este tráfico de fauna centroamericana, el cual se mantuvo hasta entrada la quinta década del siglo XX. Como bien apunta Parsons, retomando a Carr, 1954:

La especie carey tiene un ámbito muy amplio y parece que sus lugares de desove son en playas muy distanciadas unas de otras en el Mar Caribe. Por otra parte, [en el lugar conocido como] Turtle Bogue - El Tortuguero-, una lengua de 32 kilómetros de arena negra entre el Río del Tortuguero y el Río Parisima [sic] al Norte de Puerto Limón, Costa Rica, parece ser una de las últimas dos zonas de desove en gran escala de la tortuga verde del Atlántico [...]. Cada verano aparecen grandes manadas de tortugas en el Tortuguero, se acoplan cerca de la costa, y ponen los huevos en la playa. Este sector de la costa siempre ha sido un

15 Ibíd., 70.

16 Es decir, 68039 gramos, aproximadamente.

17 Libras esterlinas - dinero-, no pesaje.

18 James Parsons, «Historia de la pesca de la tortuga en el Caribe occidental», en Memorias de arrecife Tortuga. Historia natural y económica de las tortugas en el Caribe de América Central (Managua, Nicaragua: Fondo de Promoción Cultural del Banco de América, 1977b), 72.

19 Harry G. Lefever, Turtle bogue. Afro-Caribbean life and culture in a Costa Rican Village (Londres y Toronto: Associated University Presses, 1992), 54. 
lugar favorito de tortugueo de los isleños colombianos y de los indios mískitos, lo mismo que de los propios costarricenses. Actualmente está arrendado en dos lotes de 16 kilómetros por el Municipio' local — Guápiles - para voltear tortugas y recoger huevos; dichosamente las concesiones hasta ahora no han sido operadas a plena capacidad. El transporte no es digno de confianza, y los lucrativos mercados norteamericanos están muy lejos. Además, las tortugas atrapadas son normalmente hembras adultas y tan grandes y menos estimadas, como más difícilmente de manejar, que los ejemplares de 100 a 150 libras que atrapan los caimanianos en los Cayos Miskitos. En temporada, de 15 de junio a 15 de agosto, unas 2.000 tortugas hembras con peso promedio de 250 libras son volteadas por los concesionarios. La Ley dispone que esto sólo puede hacerse después que las hembras han desovado, pero los veladores' que patrullan todas las noches la playa sin que nadie los vigile, tienen mucha prisa, pues el pago es por cabeza y nadie va a saberlo. «Llevadas a Limón, las tortugas son puestas en corrales y se les da de comer bananos y hojas de banano hasta que se venden, ordinariamente a unos US \$10.00 cada una. El mejor mercado de exportación es Colón; ordinariamente unas pocas van a San Andrés». A los negros de habla inglesa les encanta especialmente la carne, que normalmente se vende a un precio ligeramente más barato que la carne de res en los mercados de Bluefields y Puerto Limón: Unas pocas son embarcadas hacia Key West, Florida, aunque no tantas como en años anteriores cuando los barcos bananeros llevaban tanques con tortugas sobre cubierta. ${ }^{20}$

La tradición extraccionista en este sector del país ha sido prolongada. Si bien a diferencia de otros lugares — por ejemplo, Abangares, Guanacaste - no fue una extracción de minerales, el rol de Tortuguero en el incipiente mercado capitalista — que se inició con el proceso de colonización del continente americano- fue marcado, entre otras razones, por el control y la apertura de vías de comunicación - naturales y artificiales-y por la comercialización intensiva y extensiva de la tortuga.

La consolidación de esas rutas del Caribe y Atlántico, respectivamente, potenció el control de las rutas marítimas y, por tanto, de los mercados de los productos americanos; desencadenando con ello lo que sería el posterior desarrollo industrial del siglo XIX e inicios del XX.

De esta manera, la tortuga fue uno de los productos estrella en ese contexto comercial, mientras las especies maderables también fueron altamente apreciadas y su extracción masiva llevó a una serie de cambios en el paisaje; así como en la vida de poblaciones tanto humanas como las faunísticas y florales de distinto tipo - ver figura 1 -.

20 James Parsons, «Historia del comercio del Carey en la Costa Caribe de Centro América», en Memorias de arrecife Tortuga. Historia natural y económica de las tortugas en el Caribe de América Central (Managua, Nicaragua: Fondo de Promoción Cultural del Banco de América, 1977c), 75, 76. Negritas añadidas. 
Figura 1. Mapa que sintetiza las rutas comerciales desde Tortuguero, se enfatizan los principales productos extraídos y explotados allí: las tortugas y la madera

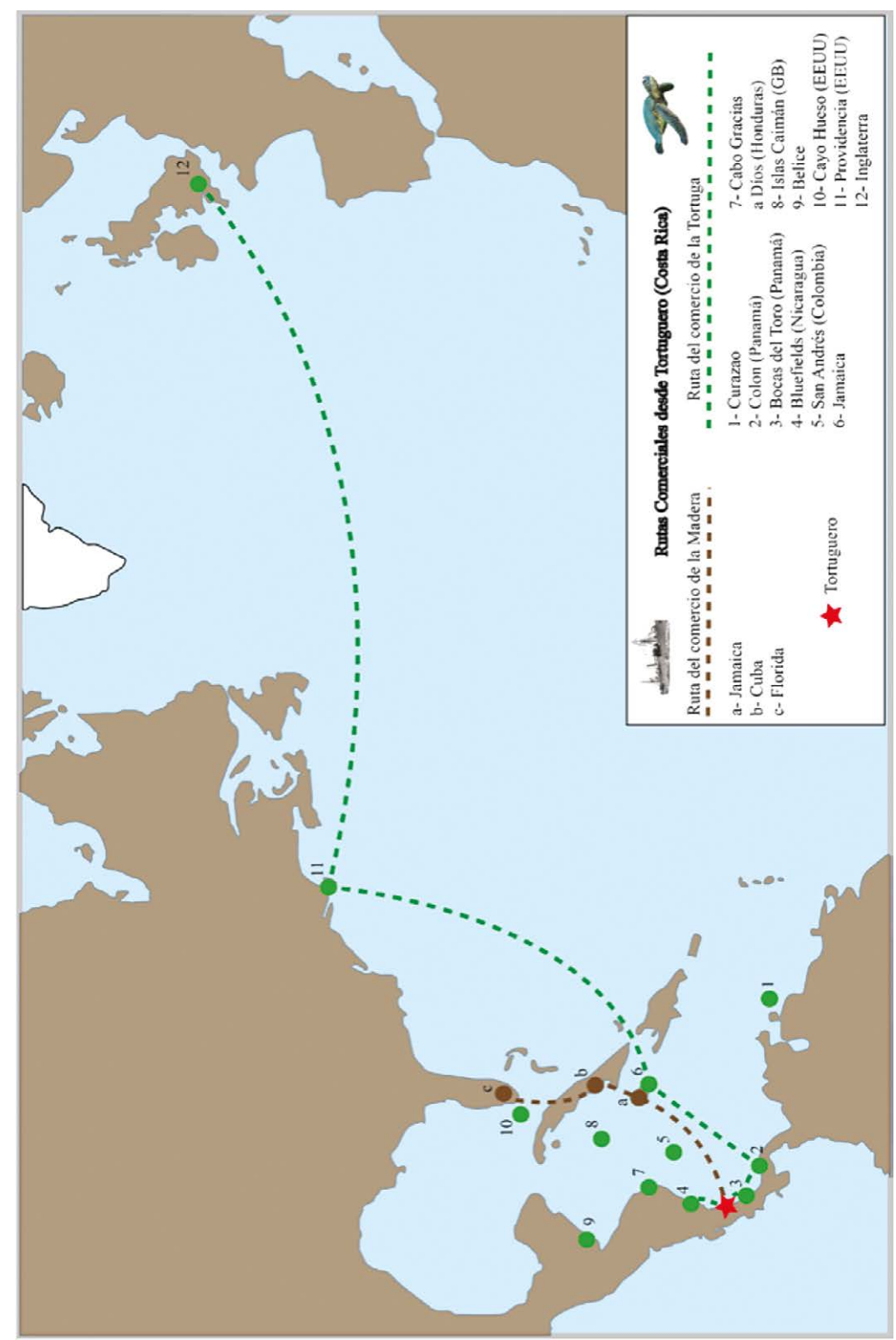

Fuente: Elaboración propia con base en datos de las obras citadas de Lefever, Parsons y Roberts. Digitalizado por el Lic. Marco Arce Cerdas, arqueólogo. 
La construcción del ferrocarril al Caribe, a finales del siglo XIX, facilitó la explotación de vastos territorios de bosques primarios y secundarios, y se involucró en estas dinámicas - de comercio global de especies - al Caribe norte de Costa Rica, así se intensifican las conocidas relaciones entre centro y periferias del capitalismo global. En particular y concerniente a la explotación de maderas en Tortuguero, entre finales del siglo XIX e inicios del XX, hubo una mayor presencia de los Estados Unidos en la esfera de interacción comercial; esto, a diferencia de lo visto para la época colonial, cuando los miskitos trataban - directa y exclusivamente - con ingleses y, en menor medida, con los franceses.

\section{Extractivismo agroforestal en Tortuguero}

La primera referencia relacionada con la extracción forestal en Tortuguero es un breve relato del sueco Carl Bovallius en la década de 1880, tras su visita a la costa este de Costa Rica. Él describe el lugar como un pequeño pueblo o colonia, habitada, en su mayoría, por trabajadores del caucho. ${ }^{21}$

Tortuguero fue por mucho tiempo un poblado con pocas personas, fundamentalmente de procedencia nicaragüense - miskitos-, quienes arribaban allí en ciertas épocas del año para la caza de tortugas. Lo usual era que dichos tortugueros se desplazaran por los ríos que estaban interconectados, según lo señaló Roberts para, «inclusive», una época relativamente tardía como 1827.

[...] hay una comunicación entre el Río Colorado y el Río San Juan — que sale del Lago de Nicaragua-, a una distancia como de 30 millas de su desembocadura, por medio del tributario conocido con el nombre de Serapiquí [sic]. Su curso en el interior es casi paralelo al del Río San Juan, y se dice que tiene muchos afluentes que tienen su origen en las montañas al sur del Lago de Nicaragua. Desemboca como a diez millas del puerto de San Juan, pero en la mayoría de los mapas aparece desembocando erróneamente a una distancia considerable al sur de su verdadera desembocadura [...]. Muchos pescadores, indios y otros, a su regreso de pescar, se detienen en esta región para recoger manatíes — vacas marinas - que abundan en el río y en un riachuelo en el extremo superior del puerto. Centenares de esos pescadores se quedan salando y ahumando la carne en Punta Arenosa - Sandy Point-, sin ser molestados por los Españoles. ${ }^{22}$

Para mediados del siglo XIX se unieron, a las visitas anuales de los miskitos, flotas de pescadores provenientes de las islas de San Andrés, Providencia y Bocas del Toro Panamá ${ }^{3}$ que, junto a los dos lugares señalados, eran parte de Colombia. Desde Bocas del Toro, los pescadores continuaron la migración hacia el norte tras las tortugas verdes y carey, hacían campamentos temporales a lo largo de la costa de Talamanca, lugares que más tarde

21 Bovallius (1977), 176, citado por Lefever, 55.

22 Orlando Roberts, Narración de los viajes y excursiones en la costa Oriental y en el interior de Centroamérica (1827) (Managua, Nicaragua: Fondo de Promoción Cultural del Banco de América, 1978), 78.

23 Parsons (1956), 39, citado por Lefever. 
se convirtieron en los poblados de Puerto Viejo y Cahuita, y continuaban su migración hasta Tortuguero. ${ }^{24}$

Los primeros asentamientos permanentes en Tortuguero se dieron a inicios del siglo XX, también con personas migrantes como su núcleo poblacional, quienes se dedicaban a la agricultura, la caza y la pesca. Asimismo, dichas personas alternaban sus actividades cuando eran contratados por las empresas madereras.

Hoy día se reconoce a ciertas familias procedentes del Caribe nicaragüense y colombiano, las cuales se desplazaron vía marítima — como se señaló, era la práctica usual desde finales del siglo XIX - y son reconocidas como las fundadoras del actual Tortuguero, esto junto a otras familias de trabajadores nicaragüenses que fueron traídas por la compañía maderera en la década de $1940 .{ }^{25}$

No se ha logrado encontrar aún registros de las primeras compañías que se dedicaron al comercio de hule y otras maderas en la zona a finales del siglo XIX e inicios del XX. No es sino hasta la década de $1940^{26}$ cuando se cuenta con documentación relacionada con la Atlantic Trading Company, aquella que construyó un aserradero y empleó a cerca de 250 personas en esa época, hecho todavía vigente en la memoria colectiva de los sujetos nativos de Tortuguero. La compañía en cuestión se dedicaba a extraer y comercializar, principalmente, madera de cedro Cedrela odorata - , laurel — Laurus nobilis—, cedro amargo - Cedrela odorata Meliaceae - y kativo - Prioria Capaifera —, entre otras especies maderables. ${ }^{27}$

El aserradero de la Atlantic Trading Company estaba localizado donde se encuentra el actual atracadero de Tortuguero. El proceso de acarreo de troncos se realizaba a través de los canales y el río hasta este sector. Los árboles eran talados en los bosques - desramados - y se tiraban al canal o río y, de ahí, aprovechando la fuerza fluvial, se direccionaban con ayuda de cables, personas y palos hasta llegar al aserradero.

En ocasiones eran troncos «sueltos», pero también se acomodaban y amarraban varios como «balsas», las cuales se navegaban. Esto dependía de la cantidad y el caudal de las aguas por época del año. Una vez en su destino, eran aserrados para hacer tablas, «tucas» y otras, las que eran transportadas - vía los canales naturales - hasta llegar a Limón. En ese puerto principal, las maderas eran comercializadas, ya sea a nivel nacional o internacional. ${ }^{28}$

Las labores para la exportación de maderas se facilitaron cuando se habilitó la navegación interna, mediante la apertura y limpieza de varios tramos que conectaban

24 Palmer (1977), 21, citado por Lefever.

25 Cloyd Taylor Martínez, vecino local de Tortuguero, comunicación personal, 2018.

26 Esta sería la última década que se estaría contemplando como parte de la arqueológica industrial, ya que cronológicamente esta incluye la temporalidad asociada a la I y II Revolución Industrial. Para la III Revolución de este tipo, que inicia aproximadamente en la década de 1950-1960, hay un cambio en los materiales utilizados - más sintéticos que de otra clase - y da inicio lo que se conocería como la informática.

27 Lefever. Micamara.es, https://micamara.es/tortuguero/.

28 Taylor Martínez, comunicación personal, 2018. 
las vías naturales - entre los canales - con Barra del Colorado y hasta Limón; hecho que hizo menos peligrosa la ruta en sí, ya que la vía marítima —que muchas personas debían tomar para salir de Tortuguero y llegar a Limón - era más extensa y penosa. Lo anterior permitió la introducción de embarcaciones más grandes que podían, inclusive, llegar a los canales —al sector del agua dulce de Tortuguero- ${ }^{29}$

A pesar de lo apuntado, es preciso recordar que, todavía a mediados del siglo XX, la zona de Tortuguero y sus alrededores seguía siendo remota. Para 1970 continuaba la tala de grandes extensiones forestales selectivas cerca de los ríos, lo cual fue controlado por la Atlantic Trading Company junto a comerciantes cubanos. El puerto de Tortuguero era ya importante hacia la década de 1950, pero después de la revolución cubana, y con la posterior desaparición de la empresa privada ligada a ese país, este comercio se detuvo. ${ }^{30}$

El espacio en el que se ubicó el poblado de Tortuguero era —en gran parte - solo un conjunto de arbustos cuando fue adquirido — en la década de 1920 - por Walton Martínez, el cual fallece entre 1940-1941. Posteriormente, esta tierra fue repartida a sus 6 hijos, quienes se establecieron y residieron en el lugar. Poco tiempo después llegó la Atlantic Trading Company que compró muchas hectáreas aledañas. Dicha compañía se retiró en el decenio de 1960 y, luego, se instauraron allí otras empresas madereras - de corta duración-que no tuvieron el mismo éxito que la primera. ${ }^{31}$

Cuando esas otras compañías madereras cerraron sus operaciones, las personas que trabajaban en ellas fueron despedidas. Los mismos exempleados madereros se quedaron en la localidad y reclamaron los terrenos, ocupando algunos las casas abandonadas de la compañía, mientras otros construyeron nuevas viviendas - con la propia madera que rescataron de los aserraderos abandonados-; a partir de ese momento, se dedicaron a prácticas tradicionales como la agricultura, la caza y la pesca. ${ }^{32}$

Como ha sucedido en otras partes del país, con la creación del Parque Nacional en 1970, surgieron algunos conflictos entre los conservacionistas y los lugareños, a quienes se les prohibió la caza de varias especies. Sin embargo, el establecimiento del Parque Nacional Tortuguero conllevó a la implementación de la industria turística, lo que ha desencadenado que la mayoría de la población actual esté vinculada a esta actividad. ${ }^{33}$

29 Taylor Martínez, comunicación personal, 2018.

30 F. R. Sluys; W. G. Van Wielemaker y J. F. Wienk, 1992, citado por A. S. van Brouwershaven, Plantation forestry in the Northen Atlantic zone of Costa Rican. Report $\mathrm{N}^{\circ} 54$, Field Report $\mathrm{N}^{\circ} 100$. Atlantic Zone Programme (Cartago, Costa Rica: Centro Agronómico Tropical de Investigación y Enseñanza; Universidad Agrícola de Wageningen; Ministerio de Agricultura y Ganadería de Costa Rica MAG, 1993), 13, disponible en: http://repositorio.bibliotecaorton.catie.ac.cr/bitstream/handle/11554/3409/Plantation_forestry_in the_northern_atlantic_zone.pdf;jsessionid=C0DF038DDA6459CCBD41F79645B5F6DD? sequence=1.

31 Lefever, 36.

32 Lefever y Cloyd Taylor Martínez, comunicación personal, 2018.

33 Taylor Martínez, comunicación personal, 2018. 
Todavía para el año 1992, Tortuguero era descrito como un pequeño pueblo — de aproximadamente 150 personas - que vivían en pequeñas casas de tablilla levantadas sobre pilotes, de las cuales cerca de la mitad tenían los techos cubiertos de hojas de palma y las otras con papel de alquitrán corrugado o zinc; había dos iglesias - una católica y una protestante-, una escuela con paredes de hormigón y techo de zinc corrugado. También se contaba con una pequeña tienda para abastecer a las personas del poblado y de las aldeas vecinas, un teléfono público y apenas dos pequeños grupos de cabañas «hoteles». ${ }^{34}$

\section{La arqueología industrial}

La arqueología es una subdisciplina de la antropología que se especializa en la investigación de las sociedades antiguas - o desaparecidas - a través de sus restos materiales. Tradicionalmente, para el caso de la arqueología local, esta se ha enfocado en el estudio de las poblaciones que habitaron el espacio que hoy conocemos como Costa Rica, previo a la conquista y la colonia. Sin embargo, la arqueología también estudia a los grupos y pueblos que vivieron posterior al siglo XVI — en este caso, los aborda desde lo que se denomina la arqueología histórica-.

El conocimiento de las personas antiguas es posible mediante el análisis de los restos materiales que aún se conservan. Así, de esta manera la arqueología busca conocer diversos ámbitos de la vida de dichas personas y, para ello, usa diferentes perspectivas teóricas y enfoques metodológicos, además de técnicas y procedimientos específicos.

La arqueología industrial, por su parte, es una de las especializaciones más recientes de la arqueología histórica y se basa en el estudio de los bienes/artefactos, los contextos y demás evidencia correspondiente con el período de desarrollo económico y tecnológico de producción industrial, el cual está relacionado con la primera y segunda revolución industrial: entre el siglo XVIII hasta inicios del XX; se excluye la tercera revolución — que dio inicios a mediados del siglo $\mathrm{XX}$ - cuando surge otra revolución tecnológica relacionada con el uso del plástico, el desarrollo informático y electrónico.

De tal modo, la arqueología industrial busca conocer la forma en que vivían las personas, las relaciones sociales que se dieron tras la producción y el consumo industrial masivo, los diversos roles sociales que se empiezan a implementar en las economías capitalistas, entre otros aspectos, visibilizando el desarrollo de infraestructuras y avances tecnológicos; así como la organización técnica y social del trabajo - incluye el comportamiento social, entornos laborales, represión, movimientos sociales, movilidad, migraciones, etc.—, al mismo tiempo que se interesa por las adaptaciones tecnológicas, políticas y económicas

34 Lefever, 35. 
de importación y exportación, el aprovechamiento y explotación de los recursos, el acceso y consumo de bienes particulares, entre otros. ${ }^{35}$

Dentro de los contextos que estudia la arqueología industrial se encuentran la infraestructura relacionada con los beneficios de café, ingenios de azúcar, ejes ferroviarios, minas, diferentes tipos de fábricas, navales, poblados de obreros, entre otros.

Lamentablemente, en muchos países latinoamericanos esta especialidad científica sigue siendo invisibilizada, porque se cree que dichas relaciones sociales se generaban solo en naciones económicamente poderosas; cuando - en realidad - los contextos sociales etiquetados como «tercermundistas»o «en vías de desarrollo» han sido claves para el desarrollo del sistema capitalista, tal y como lo conocemos históricamente y, por ende, son fundamentales para el entendimiento del desarrollo industrial global.

Por lo anterior, además del potencial de conocimiento sobre los procesos acaecidos durante la época colonial y republicana, dicha subdisciplina ha ganado espacio en diversos países, lo que ha fomentado la patrimonialización y conservación de diversos restos arqueológicos ligados a estas épocas para su visita y disfrute, principalmente, el turístico - por ejemplo, en España, Portugal, México, Brasil, Colombia, Guatemala-. No obstante, para el contexto local y al corresponder a una época más reciente, el patrimonio industrial queda fuera de la protección que brinda la legislación relacionada con la arqueología, regida por la ley 6703 ; por lo que dichos bienes sufren constantes depredaciones, vandalismo y, a la vez, están sujetos a muy poca investigación científica. ${ }^{36}$

En Costa Rica este tipo de contextos datan - fundamentalmente- del siglo XIX e inicios del XX cuando el país, posterior a su independencia, buscó hacer una importante inversión para el desarrollo de infraestructura, sus vías de comunicación y la incursión de la industria — principalmente la agraria-, lo cual queda evidenciado en haciendas, por ejemplo: Coyolar, El Molino, Agua Caliente, entre muchas otras; así como en el desarrollo de los ejes ferroviarios del Pacífico, del Caribe y los ramales generados hacia fincas que estaban produciendo diversos productos; pero, mayoritariamente, para facilitar el transporte en las plantaciones bananeras. Otros ejemplos del desarrollo industrial son el tranvía de San José- de Cartago, fábricas como la FANAL — su edificio alberga el actual Ministerio de Cultura y Juventud- y variadas estructuras como las edificaciones metálicas, la casa de la Moneda, imprentas, las minas de Abangares, etc.

35 Mónica Aguilar y Jeffrey Peytrequín, Proyecto B8-077 Arqueología Industrial, estado del arte y primer inventario nacional (San José, Costa Rica, 2018), 2.

36 Aguilar y Peytrequín, 2. 
La historia reciente de Costa Rica está permeada por las políticas de gobiernos de corte liberal que buscaron el desarrollo de los grandes territorios fuera del Valle Central, con lo cual se brindaron incentivos para la «colonización de terrenos baldíos» y lugares apartados, todo ello conexo a la producción del café y otros cultivos de consumo local; así como el auge, mediante la economía de enclave, en la producción y exportación del banano. De esta manera, se dieron concesiones en diversas partes del país con el objetivo de poblar, extender los cultivos del café, y desarrollar la infraestructura ferroviaria y vial que facilitara la exportación de dicho grano a Europa y los Estados Unidos; en ese contexto varios terrenos son dados en concesión a Mynor Cooper Keith, entre otros inversionistas tanto nacionales como extranjeros. ${ }^{37}$

Lo anterior, más la apertura comercial que se dio luego de la independencia de España, es lo que acelera la importación y producción industrial de diferentes productos, al igual que propició cambios importantes en muchas partes de Costa Rica con modelos económicos ajenos, el ingreso de mano de obra extranjera, así como la modificación en el uso del suelo; todo esto repercutió, inclusive, en la alteración de numerosos sitios arqueológicos antiguos.

La importancia del estudio que se realiza desde la arqueología industrial radica en la valorización no solo de los inmuebles, los artefactos y demás evidencia arqueológica, como parte del patrimonio de una nación, sino también por los datos científicos que se producen y permiten explicar fenómenos sociales del pasado reciente y sus repercusiones en el presente.

Como bien señala la Carta Nizhi Tagil, producida por el TICCIH —organización mundial encargada del patrimonio industrial y asesora especial de ICOMOS en temas relativos a este ámbito-:

El patrimonio industrial se compone de los restos de la cultura industrial que poseen un valor histórico, tecnológico, social, arquitectónico o científico. Estos restos consisten en edificios y maquinaria; talleres, molinos y fábricas; minas y sitios para procesar y refinar; almacenes y depósitos; lugares donde se genera, se transmite y se usa energía; medios de transporte y toda su infraestructura; así como los sitios donde se desarrollan las actividades sociales relacionadas con la industria, tales como la vivienda, el culto religioso o la educación. ${ }^{38}$

En la actualidad se cuenta con pocas investigaciones arqueológicas relacionadas con el patrimonio industrial en Costa Rica. Existen algunas declaratorias, como «Monumentos histórico-arquitectónicos», mediante la ley 7555 , que incluyen contextos industriales; aunque la fundamentación para esas declaratorias se basa, en su mayoría, en investigaciones de corte histórico y arquitectónico.

37 Antonio Castillo, La guerra del oro. Tierra y minería en Abangares (1890-1930) (San José, Costa Rica: EUCR, 2009); Watt Stewart, Keith y Costa Rica (San José, Costa Rica: ECR, 1967).

38 Comité Internacional para la Conservación del Patrimonio Industrial (TICCH) (Carta Nizhi Tagil, 2003), https://www.icomos.org/18thapril/2006/nizhny-tagil-charter-sp.pdf. 
Desde la arqueología, como ejemplos concretos y particulares, se ha hecho el registro de un contexto industrial correspondiente a la antigua hacienda El Coyolar, ubicada dentro del actual Parque Nacional Carara; esto, como parte de un trabajo final de graduación universitario ${ }^{39} \mathrm{y}$, más recientemente, se dio el primer trabajo de licenciatura enfocado en el tema arqueológico industrial. Dicho estudio se llevó a cabo en el contexto ferroviario de Turrialba —en las estaciones de Juan Viñas y Peralta-; se buscó conocer las dinámicas socioeconómicas relacionadas con ambas estaciones, así como la distribución espacial ligada al eje ferroviario y a los sistemas productivos..$^{40}$

A pesar de lo anterior, siguen siendo pocas las investigaciones desarrolladas en este campo especializado. Esa carencia de información puede estar relacionada con el desconocimiento que existe acerca de esta temática en el contexto nacional; por lo que el presente artículo busca, de forma complementaria, concientizar sobre el tema y divulgar los conocimientos adquiridos en torno al contexto arqueológico industrial en Tortuguero.

\section{Arqueología industrial en Tortuguero, Costa Rica}

En la actualidad, en el centro de Tortuguero se puede apreciar cierta maquinaria oxidada — «abandonada»-y propia de las actividades extractivistas de las compañías madereras que operaron en el lugar. Esta cultura material se ubica cerca del atracadero y se concentra en los parques de uso común en el núcleo del pueblo. Asimismo, aún se conserva el principal canal artificial que se construyó en la época de auge maderero para facilitar la salida y el tránsito fluvial de los troncos, conocido como «Canal o Caño Harold», el cual se encuentra dentro del Parque Nacional y puede ser visitado o transitado.

El sistema de canales de Tortuguero tiene una extensión de $112 \mathrm{~km}$ en línea paralela al Mar Caribe y va desde Moín hasta Barra del Colorado, su ancho varía de 7 a $150 \mathrm{~m}$ y presenta profundidades de 2 hasta $10 \mathrm{~m} .{ }^{41} \mathrm{~A}$ la vez, los canales fluctúan en su nivel — caudal- durante los meses secos, ya que sus profundidades pueden bajar hasta los $0,50 \mathrm{~m}$ provocando, ocasionalmente, botes encallados en espera de que suba la marea. ${ }^{42}$

39 Jorge Ramírez, «Hacia una adecuada gestión de los recursos arqueológicos presentes en el área protegida del Parque Nacional Carara, Costa Rica» (Tesis de Licenciatura en Antropología con énfasis en Arqueología, Universidad de Costa Rica, 2014).

40 Luis Andrés Arce, «El ferrocarril al Atlántico: distribución espacial y procesos socioeconómicos en las estaciones de Juan Viñas y Peralta (1870-1940): una aproximación desde la arqueología industrial» (Práctica dirigida de licenciatura en Antropología con énfasis en Arqueología, Universidad de Costa Rica, 2015).

41 Mediciones tomadas y corroboradas a través de la presente investigación.

42 Fanny Venegas, «Observatorio de Vida Silvestre Tortuguero. Una conexión vivencial a los contextos natural y social» (Proyecto final de graduación de Licenciatura en Arquitectura, Universidad de Costa Rica, 2013), 23. 
Figura 2. Ubicación del sitio arqueológico Tortuguero y ejemplificaciones - fotogrametrías en 3D — de su patrimonio industrial

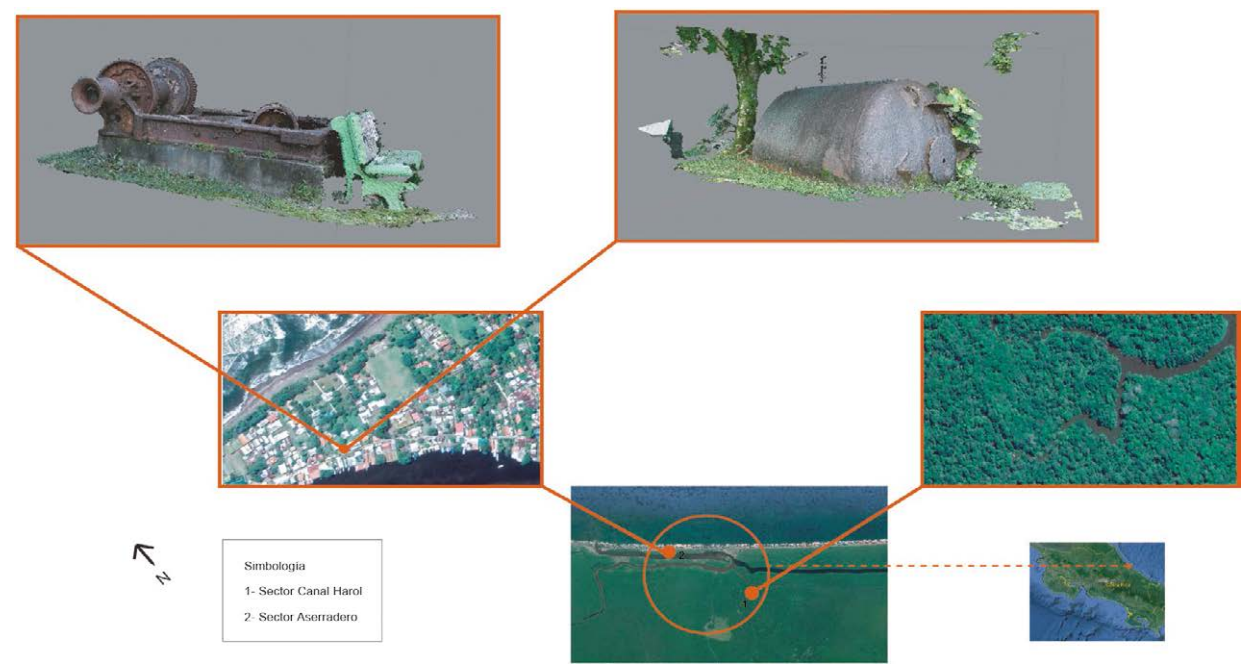

Fuente: Realizado por el Lic. Marco Arce Cerdas, arqueólogo, con base en Google maps.com 2019 y fotografías propias.

Dentro de los reconocimientos de campo realizados en el lugar, es posible identificar, al menos, dos sectores del sitio arqueológico industrial. El primero es el correspondiente al canal artificial Harold —o «Caño», como también le llaman los lugareños-; el cual posee una extensión $3500 \mathrm{~m}$, una profundidad promedio de 3,60 $\mathrm{m}$ y un ancho de $13,80 \mathrm{~m}$ - ver figura 2-. En dicho canal artificial es posible observar espacios en donde se conserva parte de los cables y las cadenas utilizadas para: a) reducir la velocidad en la que viajaban los troncos cortados sobre el río, así como b) para amontonarlos y, b) para redireccionarlos hacia los sectores de los canales y río deseados en su trayectoria hacia el aserradero - ver figura 3 -. 
Figura 3. Ubicación de los cables del sitio arqueológico industrial Tortuguero
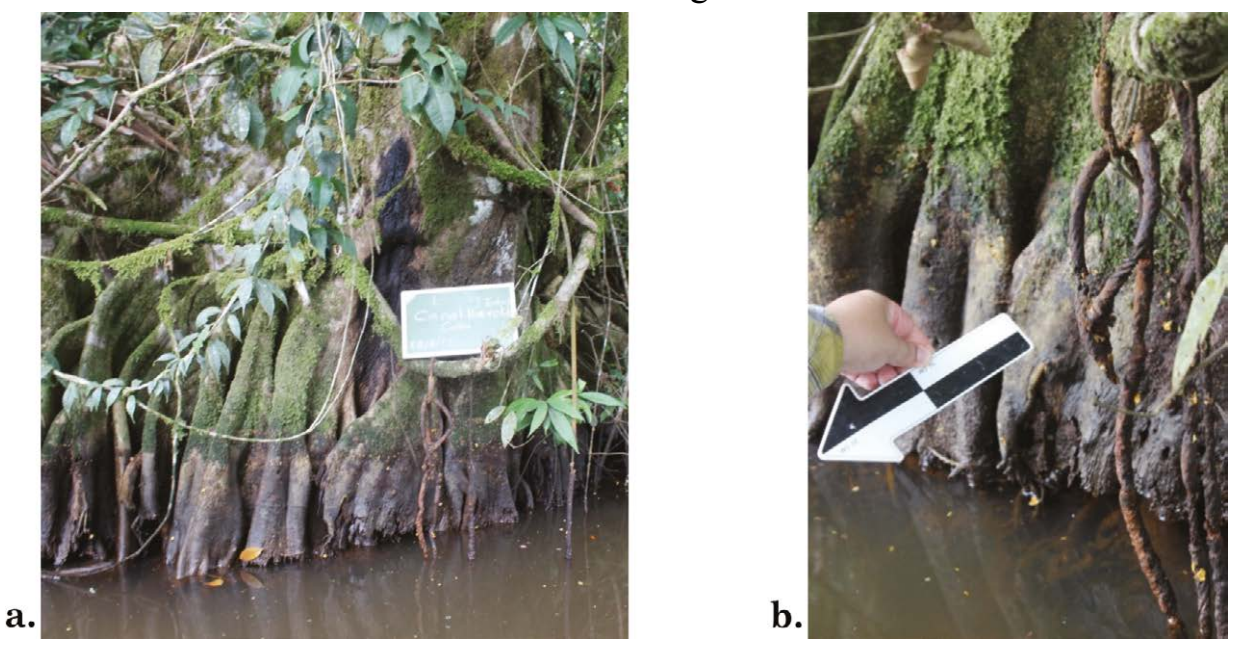

Fuente: Fotografías propias, 2018. Escala $40 \mathrm{~cm}$.

Notas: a) Los cables y cadenas se usaban para reducir la velocidad de los troncos, en su traslado fluvial, y facilitan su redireccionamiento. Estas, como se puede apreciar, están siendo absorbidas por las especies vegetales en su crecimiento; b) Detalle de los cables.

El segundo sector del sitio industrial Tortuguero corresponde al espacio del antiguo aserradero, que se ubicaba en una zona donde, actualmente, está el atracadero principal del pueblo y un parque público. Aquí, es posible reconocer varia maquinaria utilizada como: un brazo mecánico, partes de vehículos, motores, una boya - ver imagen superior derecha en la figura 2-, entre otros. Por efectos de espacio, a continuación, se pasará a discutir algunos hallazgos puntuales relativos a la cultura material industrial examinada en Tortuguero.

Entre toda la maquinaria presente - e inventariada hasta ahora - llama la atención una bomba para extraer agua de pozo. Este aparato era crucial en un espacio tan lejano y caliente; pero, a diferencia de los demás restos industriales - la mayoría de fabricación estadounidense-, esta bomba es de procedencia japonesa - ver figura 4 -

Las bombas de mano para la extracción de agua no solo eran utilizadas con aguas subterráneas, sino también se ubicaban en lugares en donde se recolectaba o almacenaba este líquido, por ejemplo, tanques con agua de lluvia, por lo que dichas bombas eran colocadas sobre una de estas fuentes de agua - ya fuera un tanque, estañón u otro- $-\mathrm{y}$, con la ayuda de una manguera, podían auxiliar en un eventual incendio. ${ }^{43}$

43 Bomba Tsuda Kibo, 1939, https://blog.goo.ne.jp/rainworld/e/44fdea80388136dc6b6ed80c577d4e5b. 
Figura 4. Bomba de mano para extracción de agua, marca Tsuda —origen japonés - , hallada en el sitio arqueológico industrial Tortuguero

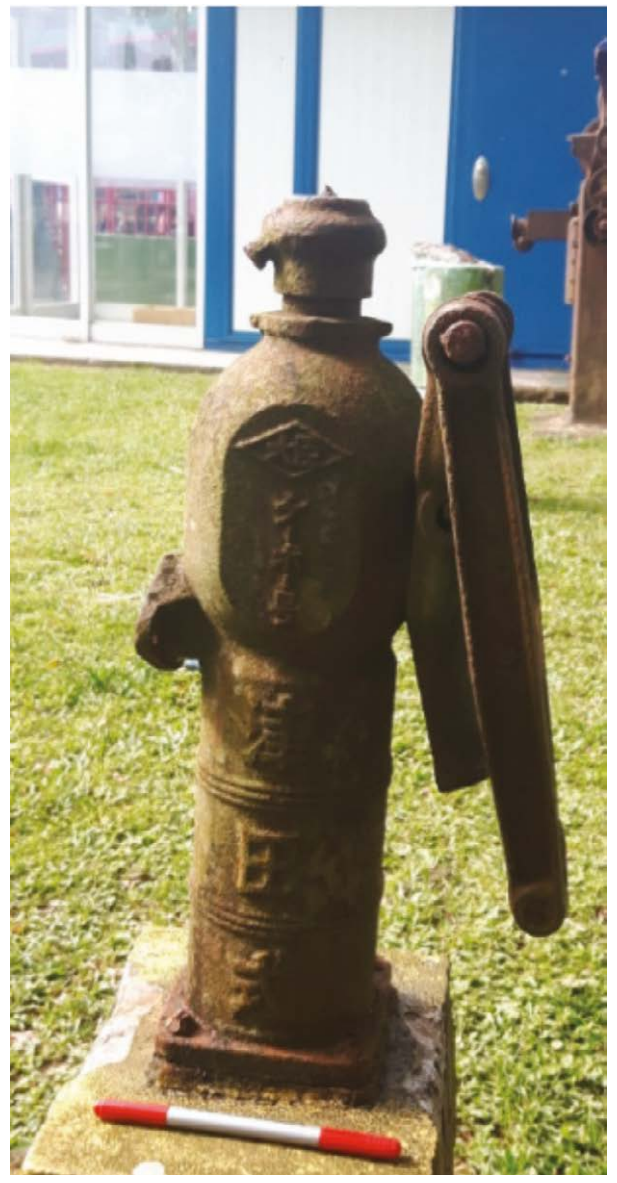

Fuente: Fotografía propia, 2018, bomba de Tortuguero.

Existía un Manual del Ministerio del Interior del Instituto de Defensa Aérea de Japón, en donde se promocionaba la bomba de mano como una protección efectiva contra incendios. Esta propaganda data de agosto de 1939 y la compañía que la fabricaba era la Tsuda Kebo. La bomba en cuestión tenía un costo — para ese momentode 1,25 yen. También, se promocionó como una bomba renovada para la protección del hogar — es decir, doméstica-, que contaba con características como ser robusta, duradera y barata; lo que hacía que, en caso de fallar, pudiese ser fácilmente reparada.

Lo interesante de este artefacto es su manufactura y propaganda en Japón, específicamente durante la II Guerra Mundial. Al respecto, los miskitos establecieron relaciones comerciales con los japoneses en épocas tardías y esta podría ser la razón que explique la presencia de esta bomba en Tortuguero, ya que se considera 
poco probable - por factores ideológicos- que fuese comprada directamente por las compañías norteamericanas que comercializaban las maderas en esos años. Por otro lado, se aclara que, aunque las industrias $T_{s} u d a$ llegaron a tener una sucursal en los Estados Unidos, esta compañía se instala en ese país posterior al conflicto bélico señalado y, por lo tanto, se sale del espectro cronológico abordado en este estudio y los artefactos manufacturados en Norteamérica no corresponderían con la fecha de producción de la bomba hallada en Tortuguero - 1939-.

Por otra parte, a diferencia de muchos aserraderos a lo largo de América Latina, en Tortuguero no se requirió desarrollar una infraestructura compleja para el traslado de los troncos, esto ya que - como fue indicado - se aprovecharon los canales fluviales para dicha empresa. Sin embargo, es en el aserradero local donde se invirtió la mayor cantidad de recursos, tal y como lo evidencian los diversos restos de maquinaria presentes en el pueblo.

Entre la maquinaria más sobresaliente — por su tamaño - se encuentra un tractor de oruga de marca Caterpillar - ver figura 5-, el cual pudo facilitar el levantamiento, acomodo y transporte de troncos, tanto en el lugar de extracción como a su llegada al aserradero de Tortuguero. La empresa Caterpillar Inc. surgió en 1925, tras la fusión de la Holt Manufacturing Co. y C. L. Best Gas Tractor Co., con sede en Illinois, Estados Unidos. ${ }^{44}$

Figura 5. Restos de maquinaria Caterpillar

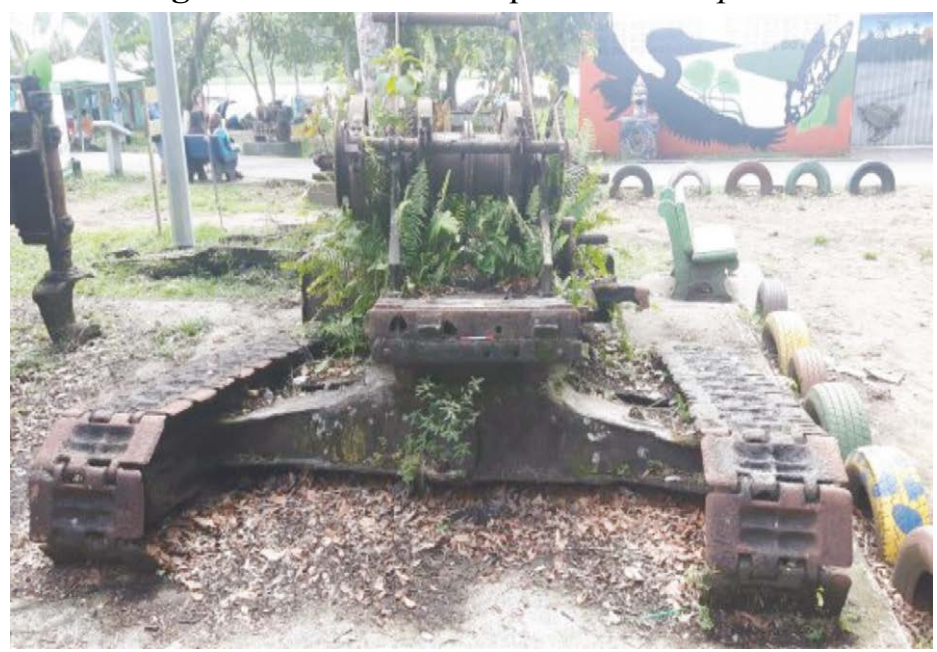

Fuente: Fotografía propia, 2018.

Notas: Resto del tractor Caterpillar en Tortuguero.

44 Blog de grúas industriales. Historia de Caterpillar Inc, 2014. https://www.gruasyequiposgarcia.com/ hisotria-de-caterpillar-inc/. 
La carga por medios mecánicos, en particular los tractores de oruga, fue introducida para la silvicultura tropical cerca de la década de 1930, debido a que era la mejor maquinaria para el desembosque de trozas grandes —obtenidas en las cortas selectivas de los grandes bosques-. Asimismo, dichas actividades exigían una gran fuerza de arrastre y una movilidad que no brindaban los antiguos arrastradores fijos.

Entre las ventajas que presentaban maquinarias como el Caterpillar D 7 - que pesa 12 toneladas - y el Allis Chalmers — con un peso mayor a 20se encuentra la presión que ejercen sobre el suelo. Esa presión era inferior a la provocaba por animales como los bueyes - los cuales generaban un peso de 1,4 $\mathrm{kg}$ por centímetro cuadrado- Así, si la maquinaria era pequeña ejercía un peso de $0,3 \mathrm{~kg}$ y si era más grande de $0,6 \mathrm{~kg}$ por centímetro cuadrado. Lo anterior se constituía en algo de mucha utilidad, más en suelos anegados como los de Tortuguero. ${ }^{45}$

Otra de las máquinas identificadas en el sector del aserradero es una fresadora Brainard. Quizás esta sea una de las máquinas más antiguas del lugar, ya que su manufactura data de finales del siglo XIX: entre 1871 a inicios del siglo $\mathrm{XX}$ - ver figura 6 -.

La fresadora es una herramienta que facilita el trabajo mecánico en diferentes materiales como la madera, el acero, el hierro y otros metales, lo que permite hacer diferentes formas en variedad, al convertir las superficies en planas, curvas, etc. Si bien se menciona que las primeras fresadoras se confeccionaron en Francia en el siglo XVIII y eran acopladas en tornos de pedal, facilitaban así el tallado de engranajes, hay cierto consenso historiográfico en señalar a Eli Whitney como el inventor de la fresadora moderna. ${ }^{46}$

Whitney fue contratado por el gobierno estadounidense en 1798 para la fabricación de 10000 rifles - los cuales para esa fecha se hacían a mano, por lo que las piezas de uno no se podían acoplar a otros- - De tal modo, el inventor ideó una máquina perfeccionada — en 1818 - que una vez fijado el modelo - o patrón de cada pieza-, se podía fabricar este de manera estandarizada. La máquina en cuestión usaba una rueda giratoria, dentada y afilada, para cortar el metal siguiendo el contorno de una plantilla; dicho modelo sirvió de base para muchas otras máquinas. ${ }^{47}$

45 Y. A. Cermak y A. H. Lloyd, «El transporte de madera apeada en los trópicos», Unasylva. Revista de Silvicultura y Productos Forestales, vol. 16, n. ${ }^{\circ}$ 4. (1962), http://www.fao.org/3/d3200s/d3200s06.htm\#iii\%20 $\% 20 \% 20$ transporte $\% 20$ por $\% 20$ agua $\% 20 \mathrm{y} \% 20$ por $\% 20$ aire:\%20operaciones $\% 20 \mathrm{de} \% 20$ carga.

46 Albert Esteves, «Dos siglos de fresadoras. Historia de la máquina-herramienta», Interempresas.net, 5 de marzo de 2003, https://www.interempresas.net/MetalMecanica/Articulos/12066-Dos-siglos-de-fresadoras.html.

47 Ibíd., 2003. 
Figura 6. Brainard Milling Machine localizada en Tortuguero

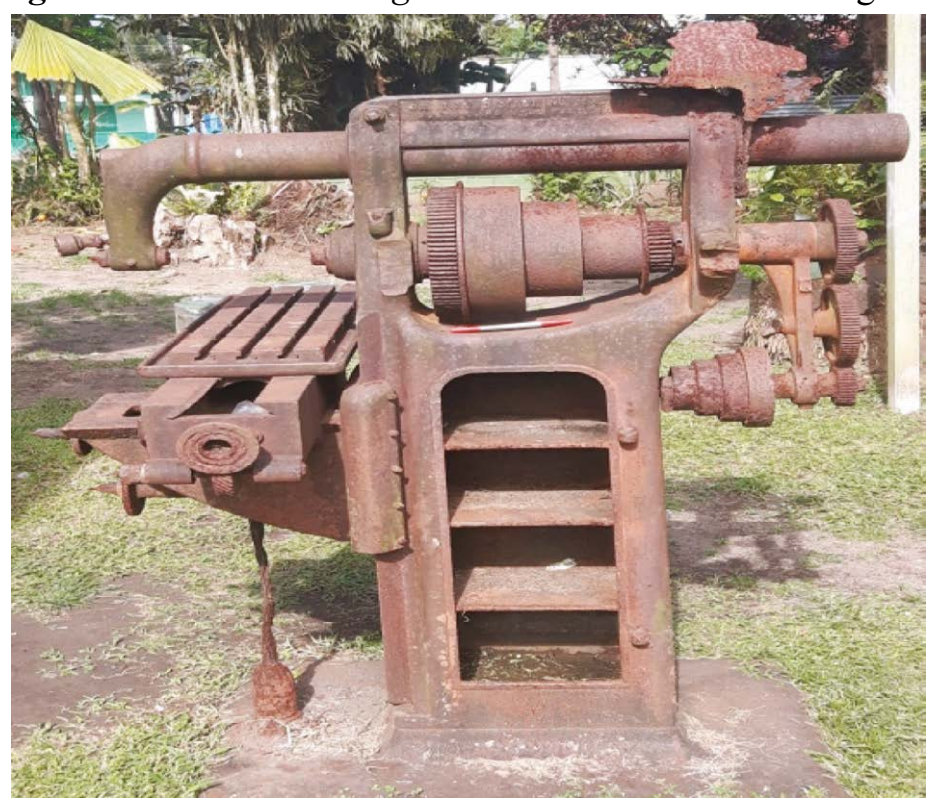

Fuente: fotografía propia.

Notas: Máquina fresadora presente en el sector Aserradero del sitio arqueológico industrial Tortuguero.

Amos Brainard era el agente de la Union Vise Co., empresa que emitía visas de patentes y, para el año 1869 , una máquina de fresado diseñada por él ya estaba en producción. Sin embargo, la fábrica se destruyó en un incendio en 1871 y dicho inventor/agente compró los activos de esta y nombró a la compañía como Brainard Milling Machine Co., nombre que mantuvo hasta cerca de 1901. El tipo de fresadora de la Brainard Milling Machine Co. encontrada en Tortuguero - ver figura 6- corresponde con propaganda de data 30 de junio de 1899. Ese anuncio de venta de maquinaria indica que la línea más completa de fresadoras y accesorios para fresado del mundo atañe a los fabricados por esta compañía; asimismo, se señalan allí los agentes asignados para su distribución en Estados Unidos, Inglaterra, Alemania, Bélgica, Dinamarca, Rusia, Suecia y Holanda. ${ }^{48}$

Los usos que permite tener una fresadora ${ }^{49}$ denotan que en Tortuguero no solo se cortaban tablas y tucas, sino que también podían hacerse acabados finos y deseados, según el tipo de mueble que se fabricara. Es muy probable que esta máquina llegara a Tortuguero con las primeras compañías madereras a inicios del siglo XX.

48 Vintage Machinery (Brainard Milling), http://vintagemachinery.org/mfgindex/imagedetail.aspx?id=6338.

49 Si se desea observar el funcionamiento de una Brainard Horizontal Mechanical Milling Machine, y para comprender parte de las funciones que debió tener esta maquinaria en Tortuguero, se recomienda al público lector remitirse a la página en línea: https:/www.youtube.com/watch?v=w-i84np2ZE8. 


\section{Algunas conclusiones sobre la arqueología industrial en Tortuguero}

El trabajo sobre la arqueología industrial desarrollado ha sido fructífero en términos de la cantidad y diversidad de información recopilada. En suma, para el sitio Tortuguero se logró el registro de parte de la maquinaria ubicada en el sector del Aserradero, esta es muy diversa, con una variedad de marcas y usos que datan de finales del siglo XIX hasta mediados del XX.

En Tortuguero los elementos arquitectónicos del aserradero no se lograron conservar, debido a que las personas de la localidad siguieron utilizando - y reutilizando - ese espacio, así como sus materiales de construcción. En cambio, los restos de la maquinaria - deteriorada por la salinidad y la intemperie - si se mantienen aún en el lugar $\mathrm{y}$, de un modo destacado, forman parte de la identidad local, de la memoria histórica que conservan, de su pueblo, las personas lugareñas.

En términos generales, tanto del estudio del sitio industrial — canales y elementos en tierra - como de los materiales analizados se observa evidencia de cultura material que se manufacturó entre 1871 a 1950, lo cual coincide, de forma directa, con la época de apogeo de inversión — en el país - de compañías extranjeras que tenían concesiones o habían comprado tierras, con el fin de obtener ganancias de su producción agrícola, forestal o minera.

Respecto a los trabajos pendientes en el contexto industrial de Tortuguero, es necesario afinar el inventario de cultura material y, sobretodo, poner en valor la historia de este sitio. Además, se deben recopilar las historias y memorias propias de la gente local relacionada con la vida previa al auge turístico/ecológico, relativas a los tiempos del extractivismo forestal; así como otras que contemplen lo cotidiano del oficio de los sujetos taladores, madereros, operarios del aserradero, sus familias y la dinámica comunal en esos tiempos — siglo XX antes de la década de 1970-.

Por su parte, es preciso documentar, en la medida de lo posible, la ruta que seguía la madera desde el aserradero o los canales de extracción, hasta el puerto de Limón. Aunque se sabe que el proceso no se podrá reconstruir de manera completa, ya que tras el terremoto de Limón —en 1991—y debido al desuso de esta ruta, en la actualidad el trayecto no es $100 \%$ navegable. ${ }^{50}$

De igual manera, hay que continuar con el registro fotogramétrico de la maquinaria presente en los parques de la comunidad — labor ya avanzada-, así como el calco de las marcas y completar su inventario. Junto a ello, se deberá realizar, en el 2020, las indagaciones acerca del funcionamiento del aserradero y el modo de vida de las personas involucradas.

50 Miguel Rodríguez, biólogo del Área de Conservación Cordillera Volcánica Central, comunicación personal, 2019. 
Todos estos datos serán fundamentales para poderle devolver esa información a la comunidad de Tortuguero; se busca, con ello, el fortalecimiento de la identidad colectiva o, cuando menos, que la gente lugareña conozcan su historia reciente. Además, se debe señalar que los remanentes del sitio arqueológico industrial están habilitados para la visitación de todo público, lo cual es fundamental para potenciar la visibilización general de la arqueología industrial en Costa Rica.

Finalmente, las investigaciones en arqueología industrial aportan al conocimiento científico social, a la valoración de un patrimonio que resulta cotidiano en los contextos en donde se ubican esos remanentes de cultura material y a la comprensión de fenómenos históricos globales que no solo incidieron en la economía de las periferias - como lo era el caso de Costa Rica dentro de la dinámica capitalista-, sino también acercarse al estudio crítico de las redes de producción, de consumo, las políticas permisivas de los Estados, el poco control de sus recursos y la conceptualización de la naturaleza como un bien inagotable; esto entre otros aspectos que inciden hasta hoy en día en nuestra concepción como país.

\section{Bibliografía}

Aguilar, Mónica y Jeffrey Peytrequín. Proyecto B8-077 Arqueología Industrial, estado del arte y primer inventario nacional. San José, Costa Rica, 2018.

Arce, Luis Andrés. «El ferrocarril al Atlántico: distribución espacial y procesos socioeconómicos en las estaciones de Juan Viñas y Peralta (1870-1940): una aproximación desde la arqueología industrial». Práctica dirigida de licenciatura en Antropología con énfasis en Arqueología, Universidad de Costa Rica, 2015.

Área de Conservación. «Proyecto Fortalecimiento del Programa de Turismo en Áreas Silvestres Protegidas. Plan de turismo sostenible Parque Nacional Tortuguero PNT». Guápiles, Limón, sin fecha. http://www.sinac.go.cr/ES/ transprncia/Planificacin $\% 20 y \% 20$ Gestin $\% 20 \mathrm{BID} / \mathrm{Gesti} \% \mathrm{C} 3 \% \mathrm{~B} 3 \mathrm{n} \% 20$ Sostenible\%20del $\% 20$ Turismo\%20en\%20ASP/Planes\%20de\%20Turismo\%2010\%20ASP/Plan\%20de\%20Turismo\%20Sostenible\%20del $\% 20$ PN\%20Tortuguero.pdf.

Bomba Tsuda Kibo, 1939. https://blog.goo.ne.jp/rainworld/e/.

Blog de grúas industriales. Historia de Caterpillar Inc, 2014. https:/www.gruasyequiposgarcia.com/hisotria-de-caterpillar-inc/.

Castillo, Antonio. La guerra del oro. Tierra y minería en Abangares (18901930). San José, Costa Rica: EUCR, 2009.

Cermak, Y. A. y A. H. Lloyd. «El transporte de madera apeada en los trópicos». Unasylva. Revista de Silvicultura y Productos Forestales, vol. 16, 4 (1962). http://www.fao.org/3/d3200s/d3200s06.htm\#iii\%20\%20\%20 
transporte $\% 20$ por\%20agua\%20y\%20por\%20aire:\%20operaciones $\% 20$ de $\% 20$ carga.

Comité Internacional para la Conservación del Patrimonio Industrial (TICCH), Carta Nizhi Tagil, sobre el patrimonio industrial (2003). https://www.icomos.org/18thapril/2006/nizhny-tagil-charter-sp.pdf.

Esteves, Albert. «Dos siglos de fresadoras. Historia de la máquina-herramienta». Interempresas.net, 5 de marzo de 2003. https://www.interempresas.net/ MetalMecanica/Articulos/12066-Dos-siglos-de-fresadoras.html.

Lefever, Harry. Turtle bogue. Afro-Caribbean life and culture in a Costa Rican Village. Londres y Toronto: Associated University Presses, 1992.

Nietschman, Bernard. Memorias de arrecife Tortuga. Historia natural y económica de las tortugas en el Caribe de América Central. Managua, Nicaragua: Fondo de Promoción Cultural del Banco de América, 1977.

Parsons, James. «Una clase de alimento tan delicioso». En Memorias de arrecife Tortuga. Historia natural y económica de las tortugas en el Caribe de América Central. Managua, Nicaragua: Fondo de Promoción Cultural del Banco de América, 1977a.

. «Historia de la pesca de la tortuga en el Caribe occidental». En Memorias de arrecife Tortuga. Historia natural y económica de las tortugas en el Caribe de América Central. Managua, Nicaragua: Fondo de Promoción Cultural del Banco de América 1977b.

. «Historia del comercio del Carey en la Costa Caribe de Centro América». En Memorias de arrecife Tortuga. Historia natural y económica de las tortugas en el Caribe de América Central. Managua, Nicaragua: Fondo de Promoción Cultural del Banco de América, 1977c.

Ramírez, Jorge. Hacia una adecuada gestión de los recursos arqueológicos presentes en el área protegida del Parque Nacional Carara, Costa Rica. Tesis de licenciatura en Antropología con énfasis en Arqueología, Universidad de Costa Rica, 2014.

Roberts, Orlando. Narración de los viajes y excursiones en la costa Oriental y en el interior de Centroamérica (1827). Managua, Nicaragua: Fondo de Promoción Cultural del Banco de América, 1978.

Stewart, Watt. Keith y Costa Rica. San José, Costa Rica: Editorial Costa Rica, 1967.

Van Brouwershaver, A. S. Plantation forestry in the Northen Atlantic zone of Costa Rican, Report N. ${ }^{\circ}$ 54, Field Report N. ${ }^{\circ} 100$. Atlantic Zone Programme. Cartago, Costa Rica: Centro Agronómico Tropical de Investigación y Enseñanza; Universidad Agrícola de Wageningen; Ministerio de Agricultura y Ganadería de Costa Rica MAG, 1993. 
Venegas, Fanny. «Observatorio de Vida Silvestre Tortuguero. Una conexión vivencial a los contextos natural y social». Proyecto final de graduación de Licenciatura en Arquitectura, Universidad de Costa Rica, 2013.

Vintage Machinery (Brainard Milling). http://vintagemachinery.org/mfgindex/ imagedetail.aspx?id $=6338$.

Woodbury, George. The great days of Piracy. Londres, Inglaterra: Elek, 1954. 
